\author{
A Thesis \\ by \\ DANIEL WALLACE HARTMAN \\ Submitted to the Office of Graduate and Professional Studies of \\ Texas A\&M University \\ in partial fulfillment of the requirements for the degree of
}

\title{
MASTER OF SCIENCE
}

$\begin{array}{ll}\text { Chair of Committee, } & \text { Richard Miles } \\ \text { Co-Chair of Committee, } & \text { Albina Tropina } \\ \text { Members, } & \text { Freddie Witherden } \\ & \text { Diego A. Donzis } \\ \text { Head of Department, } & \text { Srinivas Vadali }\end{array}$

December 2020

Major Subject: Aerospace Engineering

Copyright 2020 Daniel Wallace Hartman 


\begin{abstract}
Shell models of turbulence were used to study the effect of isotropic turbulence on the refractive index spectrum for the case of a plane wave beam propagation through turbulent flow. Existing theories of the $1 \mathrm{D}$ and $3 \mathrm{D}$ temperature spectra were shown to be inaccurate in the dissipative range of the spectrum due to unresolved scales at high Reynolds numbers. The shell model explaining the buoyancy driven turbulence was chosen as a tool to consider density changes with temperature and resolve all the range of wavenumbers up to the Kolmogorov's scale. The physical nature of the Hill bump (increase of the slope of the energy spectrum), observed in the transition region between inertial-convective and viscous-diffusive parts of the cascade was explored. It was shown that no Hill bump is formed if the sufficient number of shells is chosen to resolve all of the spectrum, but the Hill bump will appear at the additional non-compensated forcing at large wavenumbers. The calculated shell-averaged temperature spectrum was used to determine the refractive index power spectral density and to create $2 \mathrm{D}$ phase-screens for modeling the laser beam propagation in the formed turbulent flow. Verification and validation of the combined shell and aero-optics problem were done by the comparison of the irradiance profiles with the laboratory experimental data and Direct Numerical Simulations for the case of a $532 \mathrm{~nm}$ laser beam propagation in grid generated turbulence and for the case of the buoyancy driven turbulence. The turbulent velocity and temperature spectra generated by the shell model were shown to be comparable to the Modified Von-Karman theory. Good quantitative agreement with the experimental data confirmed that the main source of the laser beam distortion at considered conditions relates to the thermal distortions.
\end{abstract}




\section{DEDICATION}

I dedicate this work on behalf of my beloved Jesus, my wonderful wife to be Avery, our sweet dog

Remy, and the best advisors I could ever askfor, Dr. Albina Tropina and Dr. Richard Miles. 


\section{ACKNOWLEDGMENTS}

I would like to thank everyone who worked tirelessly on my behalf to assist me in this endeavor. Dr. Albina Tropina, Dr. Richard Miles, Dr. Diego Donzis, and Dr. Chris Limbach among many others. I would also like to thank Dr. Freddie Witherden and his contributions as a member of my committee.

I also want to recognize that this work is supported both by Texas A\&M and in collaboration with the Army Futures Command (AFC) directive. This is the beginning of what will be an extended research effort to understand all the physical phenomena that relate to the propagation of light through turbulence in our atmosphere.

I believe all of this was made possible by Jesus, the name above every other name. 


\section{CONTRIBUTORS AND FUNDING SOURCES}

\section{Contributors}

This work was supported by a committee that contains Dr. Albina Tropina, Dr. Richard Miles, and Dr. Diego Donzis of the Department of Aerospace Engineering, and Dr. Freddie Witherden of the Department of Ocean Engineering.

The experimental data used for validation comparison was developed by Dr. Chris Limbach in accordance with research done for the Army Futures Command (AFC) initiative. The direct numerical simulation results used for verification of the irradiance profiles was performed by Dr. Diego Donzis and Komal Kumari. Code for the Split-Step method written by Schmidt [20] in MATLAB was translated and adapted to python for coherence between the models.

All the other work provided in this thesis was completed by the student independently.

\section{Funding Sources}

This work was supported by internal funds provided by the Texas A\&M University Aerospace Engineering Department and the Army Futures Command (AFC) initiative. 


\section{TABLE OF CONTENTS}

\section{Page}

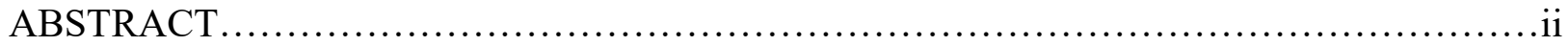

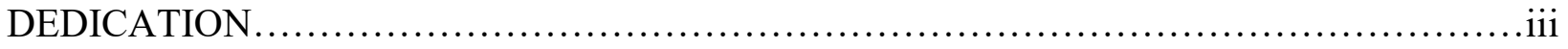

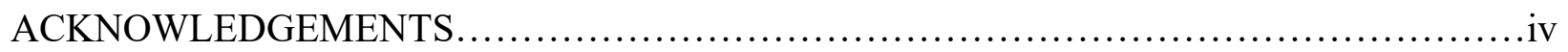

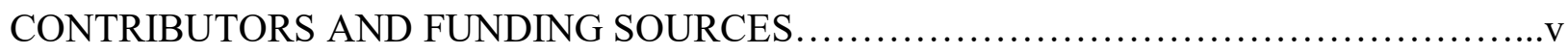

LIST OF FIGURES............................................................. vii

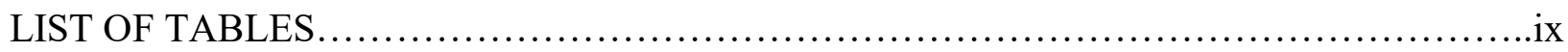

CHAPTER 1 INTRODUCTION....................................................

CHAPTER 2 THEORY OF ISOTROPIC TURBULENCE $\ldots \ldots \ldots \ldots \ldots \ldots \ldots \ldots \ldots \ldots \ldots \ldots \ldots . \ldots \ldots$

2.1 - Kolmogorov Theory.................................................4

2.2 - Buoyancy Driven Turbulence..........................................

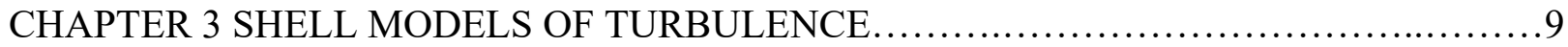

3.1 - Dyadic Shell Model of Turbulence..........................................12

3.2 - Brandenburg Shell Model of Turbulence...................................20

CHAPTER 4 LIGHT PROPAGATION IN A TURBULENT FLOW..........................23

4.1 - Tatarski Theory and the Hill Bump..........................................24

4.2 - Irradiance Patterns and Split Step Method..................................25

CHAPTER 5 REFRACTIVE INDEX SPECTRUM....................................28

5.1 - Active Scalar Plots....................................................... 28

5.2 - Buoyancy Driven Turbulence..............................................29

5.3 - Comparison to Experiments........................................... 31

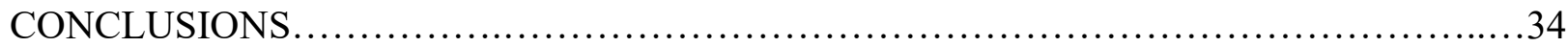




\section{LIST OF FIGURES}

FIGURE

Page

1. - Velocity Pulsation Spectrum Dependence on Time during the Energy Cascade

2. - Temperature Pulsation Spectrum Dependence on Time during the Energy Cascade .14

3. - Active Scalar Velocity Pulsation Dependence on Time during the Energy Cascade....

4. - Active Scalar Temperature Pulsation Dependence on Time during the Energy Cascade.....17

5. - Dependence of the Velocity spectrum on Reynolds Number.

6. - Dependence of the Temperature Spectrum on Prandtl number

7. - Absolute Difference in $\log \_10$ of the Temperature pulsations for $\operatorname{Re}=400, \Delta \mathrm{T}=40$,

$$
\operatorname{Pr}=0.72, \operatorname{Ri}<<1
$$

8. - Absolute Difference in $\log \_10$ of the Velocity pulsations for $\operatorname{Re}=400, \Delta \mathrm{T}=40$,

$$
\operatorname{Pr}=0.72, \operatorname{Ri}<<1
$$

9. - Energy dissipation rate dependence on time (the Brandenburg Shell Model)...

10. - Hill bump comparison to various existing similarity functions

11. - Refractive Index Pulsation Spectrum Dependence on the temperature difference .36

12. - Irradiance Profile generated using the temperature spectrum from the Dyadic Shell

Model with $\operatorname{Re}_{\mathrm{L}} \sim 400$

13. - Irradiance Profile generated using the temperature spectrum from the Brandenburg

Shell Model with $\mathrm{Re}_{\mathrm{L}} \sim 400$ 
14. - Irradiance data visual comparison to DNS and experimental measurements................31

15. $-\chi^{2}$ statistics comparison between Shell Models and Experiments...........................32 


\section{LIST OF TABLES}

TABLE Page

1. - Total Kinetic Energy as a function of time (Temperature is a Passive Scalar)..............15

2. - Time dependence of the total kinetic energy for temperature as an Active Scalar...........16

3. - Time dependence of total entropy for Temperature as an Active Scalar..................17 


\section{CHAPTER I}

\section{INTRODUCTION}

The effects of turbulence span a wide range of applications to aerospace engineering. While these effects have been studied for a long period of time, turbulent phenomena is still difficult to predict for large scale applications, for example, laser beam propagation in atmospheric turbulence. In the past, much attention has been paid to so-called aero-optical effects, connected with the performance of the laser beam systems in a turbulent media. The primary cause of aerooptical effects is the refractive index. Knowledge of refractive-index fluctuations in the turbulent flow is needed for the development of better quality and more reliable directed energy applications. Many researchers have studied the aero-optical effects trying to understand a nature of optical distortions and refractive-index fluctuations in different turbulent flows ranging from shear and boundary layers to the grid-generated turbulence [1-3]. The well-established Tatarski theory proposed a standard model of turbulent air fluctuations which was derived from the ObukhovCorrsin theory, leading to the expression

$$
\Phi(k)=0.033 C^{2}{ }_{T} k^{-\frac{11}{3}} b\left(k l_{0}\right),
$$

where $C^{2}{ }_{T}$, the second order structure function for temperature, $l_{0}$ is the inner temperature scale. In the Tatarski theory $l_{0}$ is the size of the smallest inhomogeneities or the size of the smallest turbulent eddies. It can be related to the Kolmogorov length scale by the following formula:

$$
\frac{l_{0}}{\eta}=\left(\frac{27 \Gamma\left(\frac{1}{3}\right)}{5} \frac{b\left(k l_{0}\right)}{P r}\right)^{3 / 4}
$$

where $b\left(k l_{0}\right)$ is the similarity function for the dissipative regime. It is a function of the inner temperature scale the properties of this function require that it is 1 until the dissipative regime 
begins. There are a variety of different similarity functions modeling temperature spectral behavior in the dissipative sub-range of the turbulent cascade. In this study we will show how the shell models compare to the similarity functions for different conditions.

For better understanding of turbulence effects on the laser beam propagation we provide theoretical analysis using shell models of turbulence, by which we can resolve all turbulent scales in the wave space, including inertial and dissipative sub-ranges of the turbulent cascade. While shell models have been used extensively to study both isotropic and anisotropic turbulence (hierarchical or tree shell model models) [11] there is a gap in the literature data, where the spectral data from shell models are directly linked to the simulation of the refractive index fluctuations.

This thesis work includes two shell models with various properties to study the laser beam propagation in isotropic turbulence. In both models [23], the Boussinesq approach is used, assuming the density pulsations influence only the buoyancy term. One of the shell models is a dyadic shell model. After Fourier transform of the Navier-Stokes equations to the wave space and shell averaging, the dyadic model includes the nearest neighbor shell interactions in the non-linear convective term. The other model is the Brandenburg model [23] which includes a reverse cascade and shows effects that simulate weak intermittency by adding an additional interaction between neighboring shells. Temperature and velocity pulsation spectrum data are the output of these models and are used to model the refractive index spectrum [3]. On the last step we use the Power Spectral Density (PSD) data and apply the inverse discrete and the split step method [19] to create two-dimensional phase maps and irradiance profiles and compare with the available experimental and Direct Numerical Simulation data. We explore the laser beam propagation through buoyancy driven turbulent flow, which is characterized by three non-dimensional parameters: the Reynolds number Re, Prandtl number Pr, and Richardson number Ri. 


$$
R e=\frac{u L}{v}, \quad R i=\frac{g \beta \nabla T_{e n s} L}{U_{\text {avg }}{ }^{2}}, \quad \operatorname{Pr}=\frac{v}{\alpha}
$$

The final output for this study connects these three non-dimensional parameters to a resultant irradiance profile at given aero-optical input parameters. Recent studies of atmospheric turbulence utilize the $C_{n}{ }^{2}$ structure parameter to characterize how turbulence affects the laser beam propagation. In the experimental study conducted by Y. Diskin et al. [9], the $C_{n}{ }^{2}$ values of the standard atmospheric turbulence were calculated over both a small range $(1.57 \mathrm{~m})$ and the large range (the $8 \mathrm{~km}$ ). Typical ${C_{n}}^{2}$ values for the ground to ground case are on the order of $10^{-15}$ while typical $C_{n}{ }^{2}$ values for the ground to air case is on the order of $10^{-16}$ for the majority of the path. 


\section{CHAPTER II}

\section{THEORY OF ISOTROPIC TURBULENCE}

Turbulence has been described in a variety of ways throughout history, but it was first characterized as a separate fluid dynamic behavior by Leonardo Da Vinci [11]. Since then, there were significant advancements in our understanding of turbulence phenomenon. Here we concentrated on the study of so-called homogeneous isotropic turbulence, when the statistics of turbulence is the same at any direction throughout the media.

\section{1 - Kolmogorov Theory}

Some of the most important contributions to the study of turbulence were Kolmogorov's three papers on the classification of turbulent regimes. Kolmogorov limited his view to that of isotropic homogeneous turbulence, where statistical properties of hydrodynamic quantities are uniform in any radial direction from any point selected inside the region. The first law that Kolmogorov described for full developed isotropic turbulence was the $2 / 3$ Law. It states that for any two points that are a distance $r$ apart, the mean square velocity increments are proportional to the distance $r$ between them in the $2 / 3$ power. In the turbulence theory, structure functions are often used to represent the statistical nature of turbulence. Calculation of nth order structure functions can be done by calculating the autocorrelation of the hydrodynamic quantity of interest. Thus, the velocity structure function of the second order is:

$$
D_{2}(r)=\langle v(x+r)-v(x)\rangle^{2} .
$$

For fully developed homogeneous isotropic turbulence, we can describe any independent intensive property with structure functions in this manner. Kolmogorov also proposed the law of finite energy dissipation. This states that if viscosity is the only independent variable in the 
experiment, then the energy dissipation rate depends only on the total energy deposition. With the previous laws, the so-called K41 theory of Kolmogorov [13] describes 3 additional axioms as follows:

1. In the limit of infinite Reynolds number all the possible symmetries of the Navier-Stokes equation, usually broken by the turbulent flow, are restored in a statistical sense at the small scales and away from boundaries.

2. The turbulent flow is self-similar at the smallest scales. This means that there is a unique scaling exponent for each scale.

3. The turbulent flow has a finite non-vanishing mass specific mean rate of dissipation $\varepsilon$.

According to those axioms the structure functions can be re-written as follows:

$$
D_{v}(r)=C_{v}{ }^{2} \varepsilon^{2 / 3} r^{2 / 3}
$$

where the constant $C_{v}{ }^{2}$ is the second order velocity structure function constant. It describes the strength of the turbulent cascade relative to the $2 / 3$ scaling. This formulation of the theory does not describe the effect of intermittency in turbulence, but later Kolmogorov extended the theory to include this physical phenomenon [25].

For homogeneous isotropic turbulence Kolmogorov defines the so-called inertial subrange, where the dominant turbulent behavior is driven by the kinetic energy cascade from the large-scale vortices to the small scales with the final dissipation due to viscosity in the smallest scale (Kolmogorov's scale). After transition to the wave space for the inertial subrange of 3D isotropic turbulence the kinetic energy spectrum follows the Kolmogorov scaling law. Kolmogorov also developed two hypotheses related to the similarity of scales at the smallest scale. They are as follows [25]: 
1. For the locally isotropic turbulence the distributions $F_{n}$ are uniquely determined by the quantities $v$ and $\epsilon$.

Kolmogorov defines a function by which two different scales of turbulence are described as similar. For a single scale, we have the following corresponding function:

$$
B_{d d}^{\prime}\left(r^{\prime}\right)=\beta_{d d}\left(r^{\prime}\right)
$$

By the same condition, this must be the same for all cases of locally isotropic turbulence. We can translate all the r' values into the local units as follows:

$$
B_{d d}^{\prime}(r)=\sqrt{(v \vec{\varepsilon})} \beta_{d d}(r / \lambda)
$$

$\lambda$ in this case is the Kolmogorov length scale. This is the similarity function definition for a locally isotropic flow as defined by Kolmogorov.

2. If the moduli of the vectors $y^{(k)}$ and of their differences $y^{(k)}-y^{\left(k^{\prime \prime}\right)}\left(\right.$ where $\left.k \neq k^{\prime}\right)$ are large in comparison with $\lambda$, then the distribution laws $F_{n}$ are uniquely determine by the quantity $\bar{\varepsilon}$ and do not depend on $v$.

Once again, the epsilon value represents the energy dissipation rate, and when the r' values are large relative to the Kolmogorov length, the following relation holds:

$$
B_{d d}^{\prime \prime}\left(r^{\prime \prime}\right)=\beta_{d d}\left(r^{\prime} / k^{3}\right)
$$

This formulation of the similarity function is critical to understanding where the inertial subrange breaks down and the theory behind the energy cascade.

This theory is well studied and verified for the isotropic and fully developed turbulence. Experiments have been done to advance the understanding of the impact of isotropic turbulence 
on aero-optical effects. In a study conducted by Sawford et al. [5] authors studied the dependence between Reynolds number and the relative dispersion statistics in isotropic turbulence using Direct Numerical Simulation up to Taylor scale Reynolds number $\mathrm{Re}=650$. The Taylor scale is the scale at which the viscous effects begin to dominate the energy cascade. It was shown that there are two regions from $\operatorname{Re}=38-240$ and $\mathrm{Re}=240-650$ where there was a different rate of the relative differential velocity changes with time. This gives us an idea about what a range of Taylor scale Reynolds numbers might be for aero-optics in a test facility to get a transition to the inertial subrange of the turbulent cascade. The influence of Taylor scale Reynolds number on the preferential concentration of heavy particles in turbulent flows was studied by Obligado et al. [6]. The corresponding range of Taylor scale Reynolds numbers is $230-400$ which falls roughly within the range described in [5]. This range falls into agreement with experiments and simulations done by our research group later in this thesis. Depending on the size of the grid that is used in experiments, we scaled this expected range to our model. Note that the authors of the paper [6] stated that the standard deviation of the inverse concentration field increases with decreasing Reynolds number. In general, once a method for the turbulence generation is identified, we can expect a certain Reynolds number range and inertial scale lifetimes. We consider that compressibility effects are small in the conditions of the experiment, and concentrated on the aerooptical phenomena in a fully developed isotropic turbulence in the incompressibility limit.

\section{2 - Buoyancy Driven Turbulence}

Buoyancy driven turbulence is turbulence in which the dominant forcing term is a function of the ensemble averaged temperature fluctuations caused by the gravity force. Since temperature variations are directly linked to density pulsations through the ideal gas law, there is a dilemma if it is possible to use the incompressible fluid approach. This issue was firstly addressed by 
Boussinesq [24], His main assumption was to take into account only density changes due to temperature variations in the gas state equation and in the gravity force but neglect density changes in the continuity equation, thus still using incompressible fluid approach. 


\section{CHAPTER III}

\section{SHELL MODELS OF TURBULENCE}

The Navier-Stokes equations are the governing equations for all hydrodynamically driven flows. Since these equations are so general, they are difficult to solve, especially when all of the turbulent scales must be resolved. As a result, it is instructive to study these equations in the wave-space, and that is where the shell models come in.

We begin with the Navier-Stokes equations in the form shown below for the incompressible fluid:

$$
\begin{gathered}
\left(\frac{\partial}{\partial t}-v \nabla^{2}\right) \boldsymbol{u}=-(\boldsymbol{u} \cdot \nabla) \boldsymbol{u}-\frac{1}{\rho} \nabla P+F, \\
\nabla \cdot \boldsymbol{u}=0,
\end{gathered}
$$

Applying the Fourier Transform and averaging on the octave of wave numbers results in thefollowing system of equations (7) in the wave-space for the Fourier amplitudes of the velocity

$$
\begin{gathered}
\boldsymbol{u}(\boldsymbol{t}, \boldsymbol{k})=\boldsymbol{u}_{\boldsymbol{k}}=\left\langle\sum_{\frac{k}{\sqrt{2}} \leq\left|k^{\prime}\right| \leq \sqrt{2} k} v_{j}\left(t, k^{\prime}\right) v_{j}\left(t,-k^{\prime}\right)\right\rangle \\
\frac{\partial \boldsymbol{u}_{\boldsymbol{k}}}{\partial t}=\left(\mathbb{I}-\frac{\mathbf{k k}}{k^{2}}\right) \sum_{\boldsymbol{p}+\boldsymbol{q}=\boldsymbol{k}} i\left(\boldsymbol{k} \cdot \boldsymbol{u}_{\boldsymbol{p}}\right) \boldsymbol{u}_{\boldsymbol{p}}-v k^{2} \boldsymbol{u}_{\boldsymbol{k}}+\boldsymbol{F}_{\boldsymbol{k}},
\end{gathered}
$$

The next step approximates the non-linear term $(\vec{v} \cdot \nabla) \vec{v}$ and interaction between different shells in the wave space, which is different depending on the shell model chosen. That results in the equation (11) for the Fourier amplitudes of the velocity, which is based on the nearest neighboring shell interactions.

$$
\frac{\partial u_{n}}{\partial t}=k_{n} \sum_{\ell, m} A_{\ell, m} u_{\ell} u_{m}-v k_{n}{ }^{2} u_{n}+F_{n} .
$$


In the equation above, 1 and $\mathrm{m}$ are the same size of $\mathrm{n}$ which is the number of shells in the system. The A matrix represents all the interactions that are possible between any set of shells. It would be mathematically possible to consider the effect of the first shell and the last shell by using this approach. Because these long-range interactions are not physical, the study of shell models is often centered around $\mathrm{n}$ where we consider the interactions between nearest neighbor shells. We can choose a simple dyadic model for this translation:

$$
\text { for } A=\left[\begin{array}{cccc}
1 & 0 & 0 & 0 \\
0 & 1 & -2 & 0 \\
0 & 0 & 1 & -2 \\
0 & 0 & 0 & 1
\end{array}\right] \text { and } n=4 \rightarrow C(n)=u_{n-1}{ }^{2}-2 u_{n} u_{n+1}
$$

The example above is a simple case, where the values at the boundary are zero. We can translate the matrix into an $\mathrm{n}$ dependent form because we recognize a pattern in the A matrix. If the dependence on $\mathrm{n}$ asks for a value outside of the matrix, say at the boundary, then that is resolved in the numerical scheme rather than changing the matrix to be larger or smaller. It will be shown later, but this $\mathrm{C}(\mathrm{n})$ function is our cascade term in the dyadic shell model.

With these shell models we can study a few different properties of the turbulent energy cascade. Since we have three non-dimensional parameters that serve as an input to the model, determining the exact effect of changing these parameters is critical. Furthermore, the nature of the Hill bump, a change of the slope in the kinetic energy spectrum at the end of the inertial subrange, will also be examined. Finally, the effects of weak intermittency on the energy cascade will be studied in the Brandenburg model. The initial amount of energy present in the system will be the same and it was taken from the experiments data conducted in our lab. The theory describing uniqueness and existence of the solution for the shell models was proposed by Cheskidov [4]. Specifically, Theorem 4.1 from the paper [4] states. 
Theorem 4.1. For every $u^{0} \in H$ and $g \in H$, there exists a solution of the dyadic shell model with $u(0)=u^{0}$. Moreover, the energy inequality

$$
|u(t)|^{2}+2 v \int_{t_{0}}^{t}\|u(\tau)\|^{2} d \tau \leq\left|u\left(t_{0}\right)\right|^{2}+2 \int_{t_{0}}^{t}(g, u(\tau)) d \tau
$$

holds for all $0 \leq t_{0} \leq t, t_{0}$ a.e. in $[0, \infty)$.

This shows that for any initial conditions, the energy must dissipate to the smallest scales unless the viscosity is 0 . It also states that for any initial conditions, the solution of the shell model exists and it is unique. With this basis, we build the following set of initial conditions for shell models:

$$
\begin{aligned}
& \boldsymbol{u}_{\mathbf{0}}=\left[0, u_{0}, 0,0,0,0, \ldots, 0\right], \\
& \boldsymbol{\theta}_{0}=\left[0, \theta_{0}, 0,0,0,0, \ldots, 0\right] .
\end{aligned}
$$

The length of the velocity and temperature pulsations vectors is $N$, which is the number of shells. The minimum number of shells depends on the Reynolds number and can be determined by the following formula:

$$
N_{\text {min }}=\log _{2}\left(R e^{\frac{3}{4}}\right)+10 .
$$

This formula only holds if the width of the wave space partitioning is 2 , which we will use for the remainder of this study. Furthermore, here we are relating the characteristic physical length scale to the largest scale size in a wave space. The initial conditions must also be constructed so that an inertial subrange develops during the cascade.

We have now described all the fundamental input parameters for each model and the theory that supports these choices. The choices for each model input parameter were made to bring the shell model behavior as close to the experimental setup and DNS as possible. There are small differences in the theory for each model and those will be further discussed in each of the following subsections 


\section{1 - Dyadic Shell Model of Turbulence}

Non-linear convective term in this model includes only interaction between two nearest shells and the resulting model equations for Fourier amplitudes of velocity $u_{n}$ are as follows

$$
\frac{\partial u_{n}}{\partial t}=k_{n}\left(u_{n-1}^{2}-2 u_{n} u_{n+1}\right)-v k_{n}^{2} u_{n}+F_{n}
$$

Following the same approach equations for the Fourier amplitudes of temperature pulsations have a form

$$
\frac{\partial \theta_{n}}{\partial t}=k_{n}\left(u_{n-1} \theta_{n-1}-2 u_{n} \theta_{n+1}\right)-v k_{n}^{2} \theta_{n}+B_{n}
$$

For the buoyancy driven turbulence equations (14)-(15) can be re-written as follows

$$
\begin{gathered}
\frac{\partial u_{n}}{\partial t}=k_{n}\left(u_{n-1}{ }^{2}-2 u_{n} u_{n+1}\right)-v k_{n}{ }^{2} u_{n}+\alpha g \theta_{n} \\
\frac{\partial \theta_{n}}{\partial t}=k_{n}\left(u_{n-1} \theta_{n-1}-2 u_{n} \theta_{n+1}\right)-v{k_{n}}^{2} \theta_{n}-\frac{d T}{d z} u_{n}
\end{gathered}
$$

A system of ordinary differential equations (16)-(17) are solved with the LSODE method through the solve_ivp function in Python.

Dependence on time was first shown to verify theorem 4.1 described in section 3.0. We want to make sure that the results that we get are verified by this theorem. This means that we should see a development of the cascade as time progresses. For the case where temperature is modeled as a passive scalar (the last term in the equations (17) is not included), we can guarantee this behavior. However, when the temperature pulsations are modeled as an active scalar (the last term in the equations (17) is included), the temporal dynamics will depend on the strength of the thermal source. 
Below is the time dependent velocity pulsation spectrum and the temperature pulsation spectrum when temperature is modeled as a passive scalar. In both plots, initial values of $u_{0}$ and $\theta_{0}$ are equal to 40 .

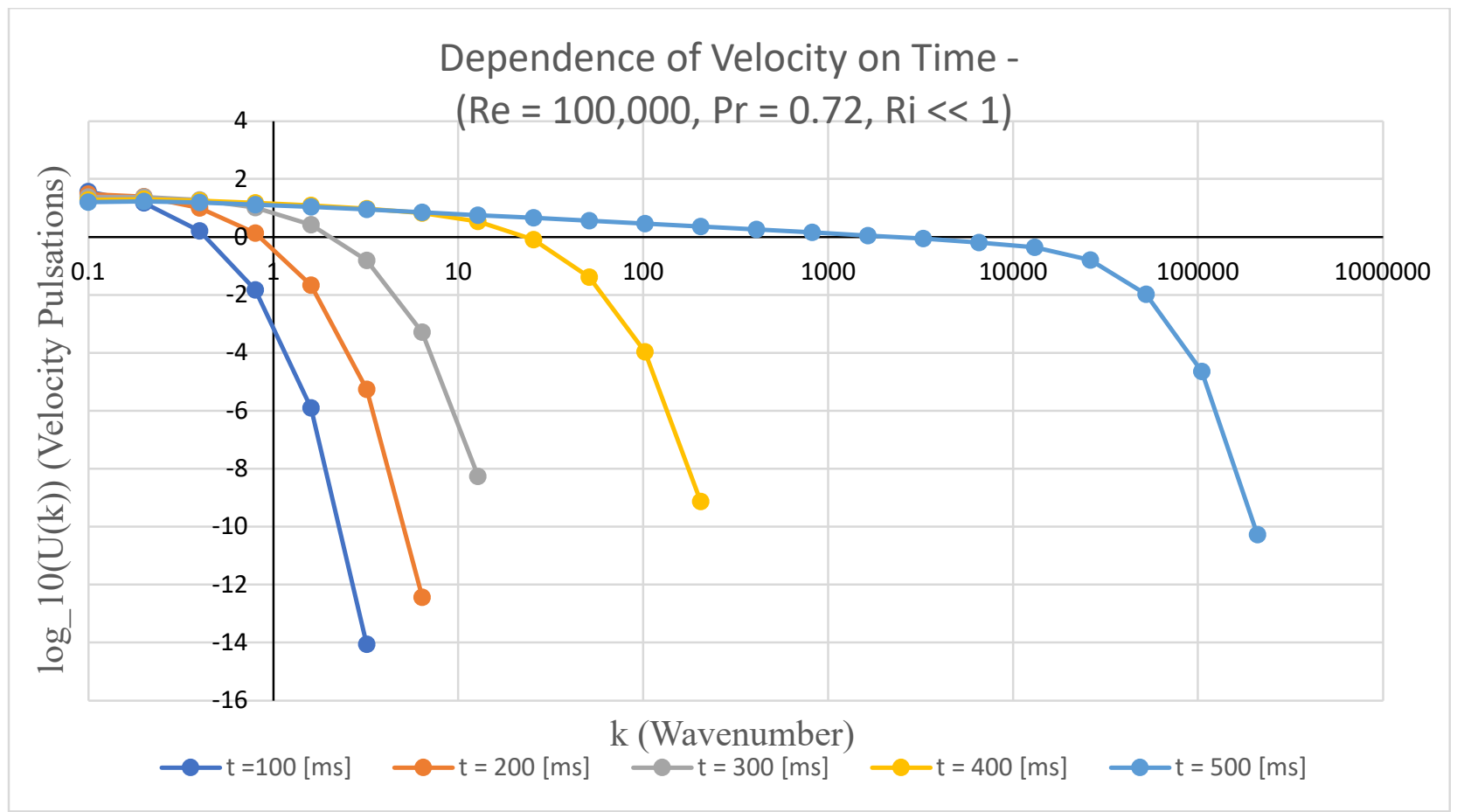

Figure 1. - Velocity Pulsation Spectrum Dependence on Time during the Energy Cascade. 


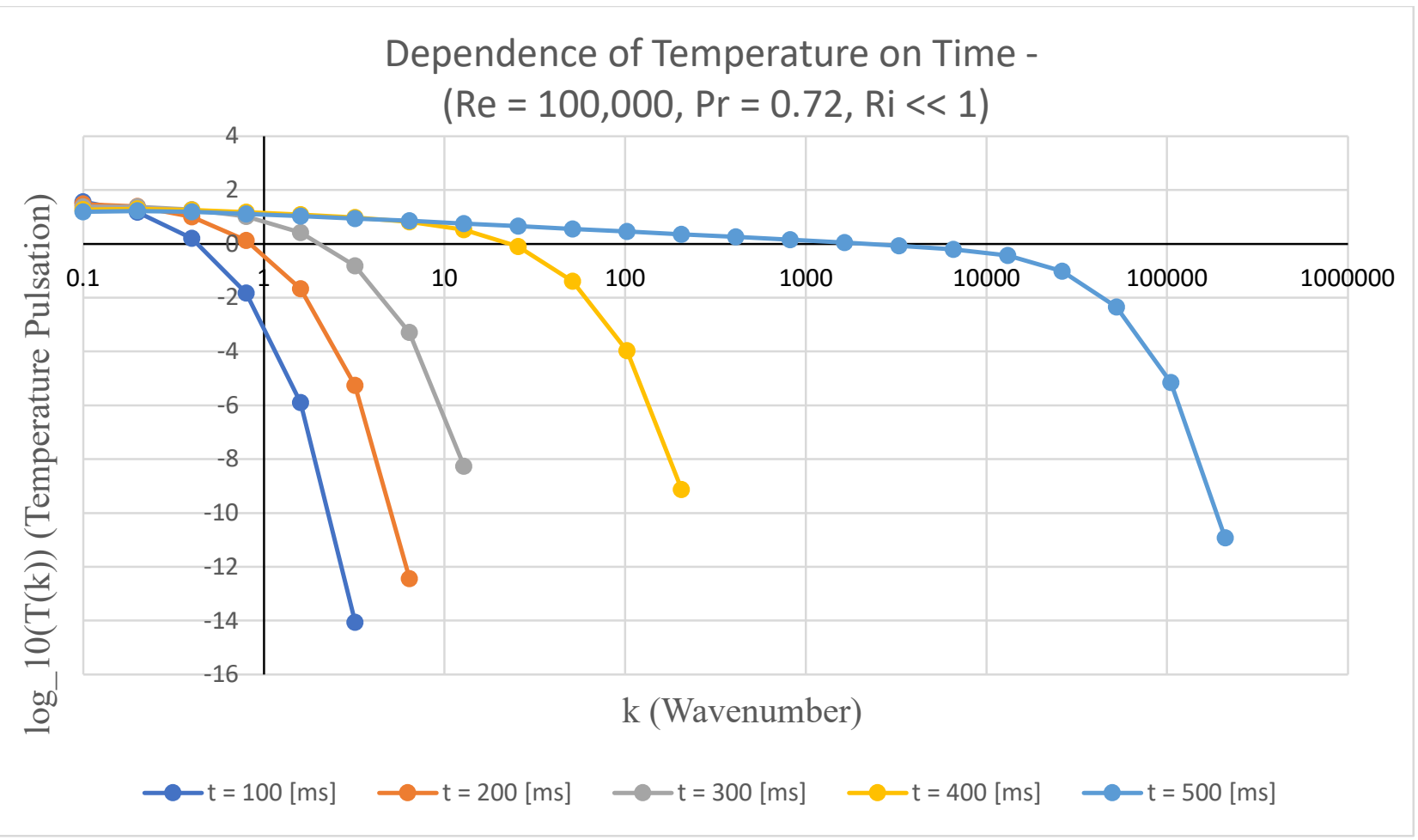

Figure 2. - Temperature Pulsation Spectrum Dependence on Time during the Energy Cascade.

This shows the behavior of the energy cascade with respect to time frame from $t=0.1$ to $t=0.5$

[s]. It can be seen in the charts above that this period is critical to the development of the inertial subrange. The range of time where the effects of the cascade are relevant is related to the viscous dissipation rate, the thermal diffusivity, and the number of shell interactions considered. The other important effect displayed is the loss of the kinetic energy through viscous dissipation predominantly in the dissipative regime. While this is difficult to see directly, we can compare the sum of all the energy values from $\mathrm{t}=0.1$ to 0.5 using a discrete integration technique in the form of a sum.

$$
E_{\text {total }}=\sum_{i}^{N} \frac{u_{i}^{2}}{k_{i+1}-k_{i}}
$$

The expression (18) was derived via dimensional analysis, and the energy spectrum can be tabulated for each time in the following way. 


\begin{tabular}{|c|c|c|c|c|c|}
\hline & $\mathrm{t}=0.1[\mathrm{~s}]$ & $\mathrm{t}=0.2[\mathrm{~s}]$ & $\mathrm{t}=0.3[\mathrm{~s}]$ & $\mathrm{t}=0.4[\mathrm{~s}]$ & $\mathrm{t}=0.5[\mathrm{~s}]$ \\
\hline$E_{\text {total }}$ & 14841.07 & 12294.31 & 9446.53 & 6800.02 & 4813.20 \\
\hline
\end{tabular}

Table 1. - Total Kinetic Energy as a function of time (Temperature is a Passive Scalar)

It is seen from the Table 1, that the total energy decreases with time as the theory predicts showing that our solutions satisfy main properties of the turbulent cascade model. It is worth noting that the total energy is affected by the forcing terms in the equation. Although the buoyancy is considered here, it is relatively small in our conditions due to the low value of the thermal expansion coefficient for air. As a result, this force has a little effect on the cascade. Furthermore, the statistical properties of turbulence do not change when the buoyant force is included. However, for the case when turbulence is driven by buoyancy the inclusion of the active scalar term in the Eq.14 affects the temperature spectrum cascade. The following plots show this effect:

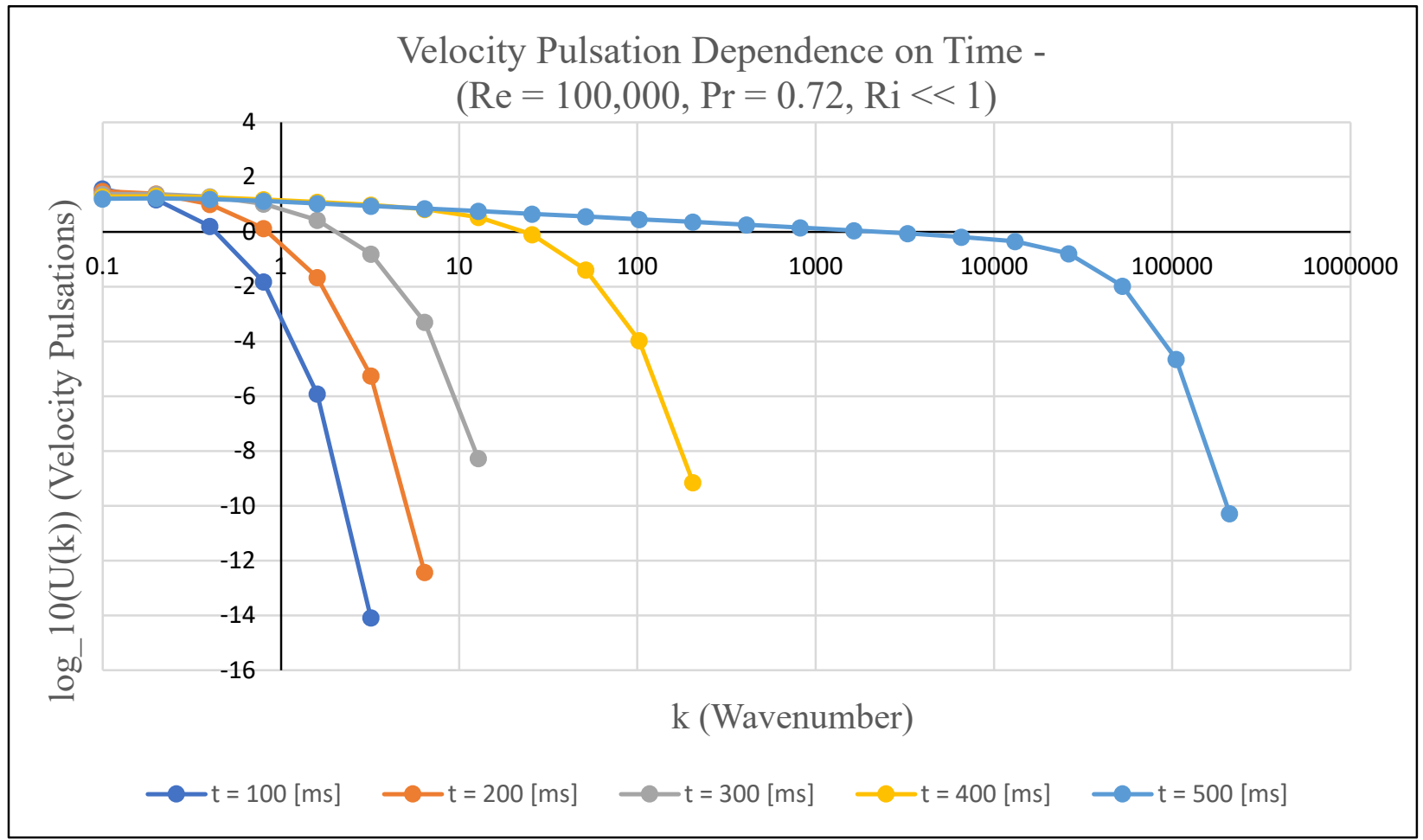

Figure 3. - Active Scalar Velocity Pulsation Dependence on Time during the Energy Cascade 
The velocity pulsations profile shown is not different than the passive scalar term. This is because the temperature gradient acts only on the buoyancy term in the hydrodynamic model and this is small in our case. The chart below shows that for all active scalar magnitudes, the total kinetic energy evolves in the same way.

\begin{tabular}{|l|c|c|c|c|c|}
\hline & $\mathrm{t}=0.1[\mathrm{~s}]$ & $\mathrm{t}=0.2[\mathrm{~s}]$ & $\mathrm{t}=0.3[\mathrm{~s}]$ & $\mathrm{t}=0.4[\mathrm{~s}]$ & $\mathrm{t}=0.5[\mathrm{~s}]$ \\
\hline$K E_{\text {total }}(18[\mathrm{~K}])$ & 14841.07 & 12294.31 & 9446.53 & 6800.02 & 4813.20 \\
\hline$K E_{\text {total }}(30[\mathrm{~K}])$ & 14841.07 & 12294.31 & 9446.53 & 6800.02 & 4813.20 \\
\hline$K E_{\text {total }}(40[\mathrm{~K}])$ & 14841.07 & 12294.31 & 9446.53 & 6800.02 & 4813.20 \\
& & & & & \\
\hline
\end{tabular}

Table 2. - Time dependence of the total kinetic energy for temperature as an Active Scalar

The units for the kinetic energy shown in the table above are shell averaged (arbitrary units). This means that we cannot say if they line up with MKS or CGS units. What we see in the table is that there is no recognizable loss or gain of the energy due to the change in buoyancy with temperature. However, the entropy has increased, and the system is losing energy faster than previously. The entropy of the system is also given by the $5 / 3$ law and it is calculated with the temperature pulsation spectrum described by the following entropy relation:

$$
S_{\text {total }}=\sum_{i}^{N} \frac{\theta_{i}{ }^{2}}{k_{i+1}-k_{i}} .
$$

Table 2 shows the total entropy for each of the cases considered.

\begin{tabular}{|l|c|c|c|c|c|} 
& $\mathrm{t}=0.1[\mathrm{~s}]$ & $\mathrm{t}=0.2[\mathrm{~s}]$ & $\mathrm{t}=0.3[\mathrm{~s}]$ & $\mathrm{t}=0.4[\mathrm{~s}]$ & $\mathrm{t}=0.5[\mathrm{~s}]$ \\
\hline$S_{\text {total }}(18[\mathrm{~K}])$ & $116,354.01$ & $260,147.59$ & $386,929.88$ & $457,233.49$ & $481,323.16$ \\
& & & & & \\
\hline
\end{tabular}




\begin{tabular}{|c|c|c|c|c|c|}
\hline$S_{\text {total }}(30[\mathrm{~K}])$ & $237,457.17$ & $602,421.15$ & $944,653.03$ & $1,149,203.79$ & 1.232 .188 .24 \\
\hline$S_{\text {total }}(40[\mathrm{~K}])$ & $371,026.82$ & $995,839.02$ & $1,596,463.60$ & $1,965,206.47$ & $2,122,640.36$ \\
\hline
\end{tabular}

Table 3. - Time dependence of total entropy for Temperature as an Active Scalar

This shows that we should expect to see a larger effect on the temperature pulsations spectrum with the inclusion of the active scalar term.

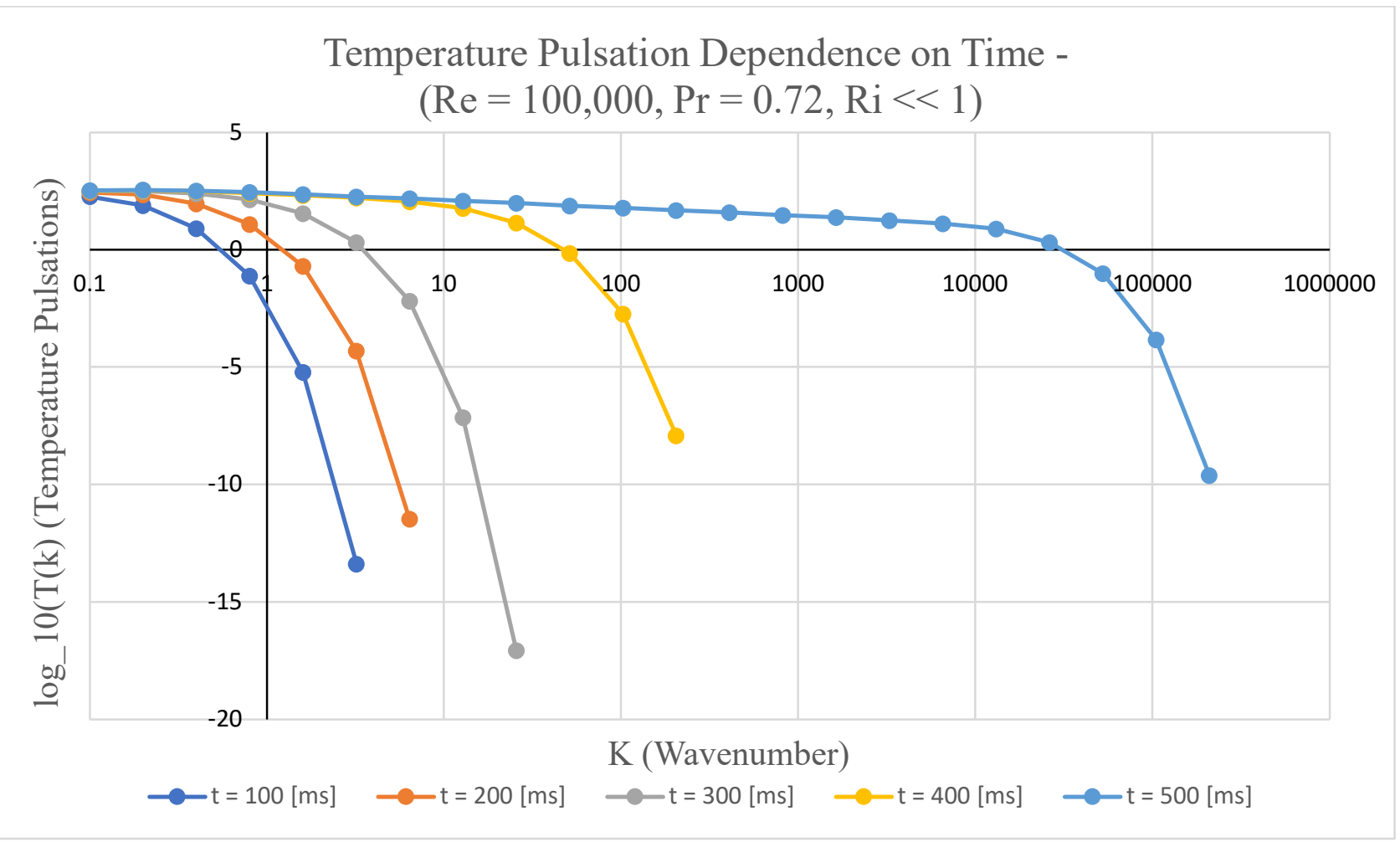

Figure 4. - Active Scalar Temperature Pulsation Dependence on Time during the Energy

Cascade

The model includes thermal diffusivity, but the active scalar term changes the temporal rate of temperature pulsations to a greater degree, as seen in Fig.4. Literature data have confirmed that the coupling between temperature and velocity spectrum through buoyancy with small temperature gradients $(40[\mathrm{~K}]$ or less) are negligible. However, the actual effect of the temperature gradient is 
seen directly in density pulsations and other physical effects such as total entropy in a wave space. Summarizing all above we have verified that the fundamental properties of the turbulent cascade are retained in this shell model.

The impact of Reynolds number on the cascade development is critical to understanding the physical structure of turbulence in applications. We tested a large range of Reynolds numbers, keeping constant both the Prandtl number, and the Richardson number.

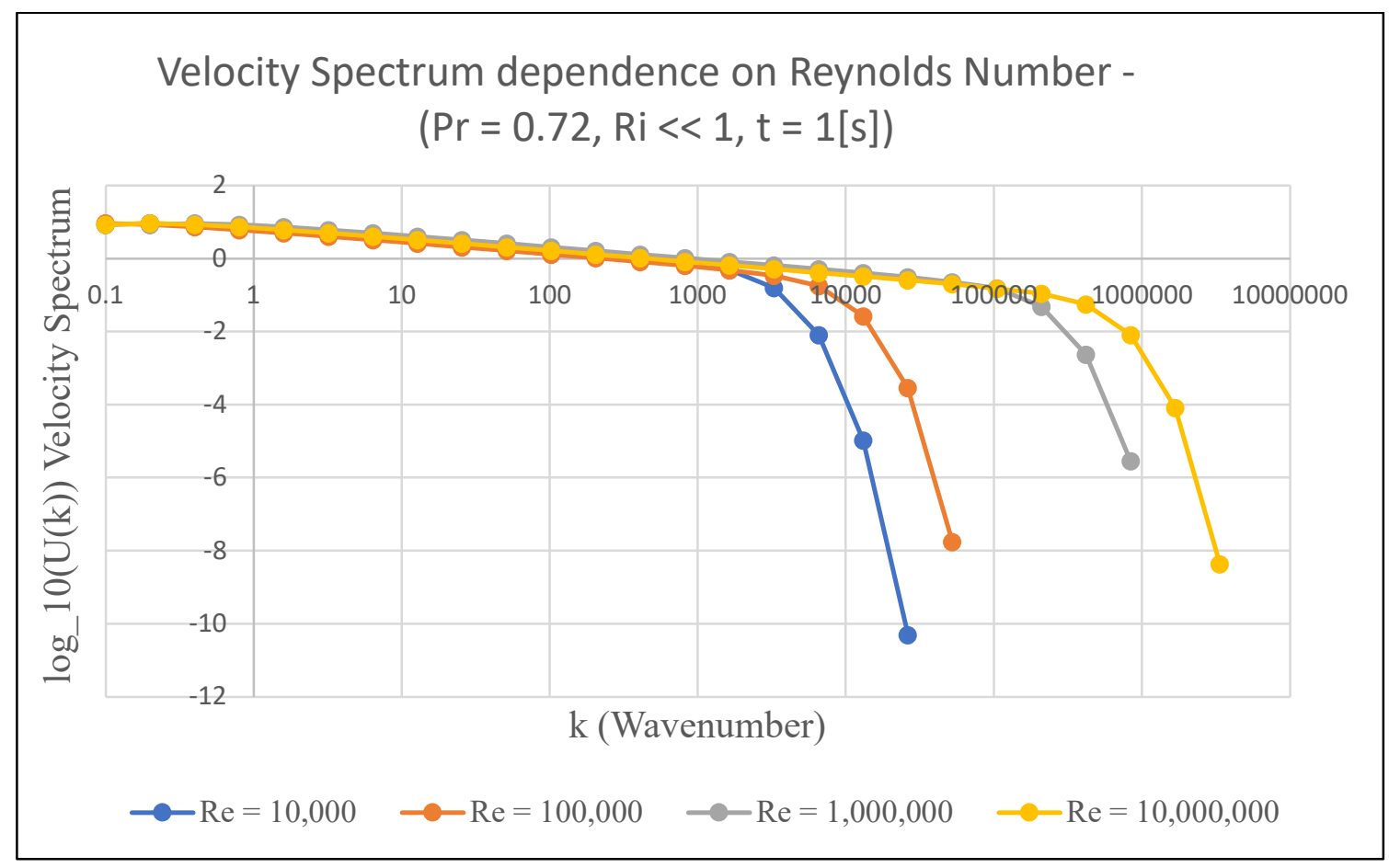

Figure 5. - Dependence of the Velocity spectrum on Reynolds Number

Fig. 5 presents the velocity spectrum at different Re numbers. It is seen that the increase in the Reynolds number directly influences the inertial subrange, causing the increase of the number of shells with Kolmogorov's scaling. 


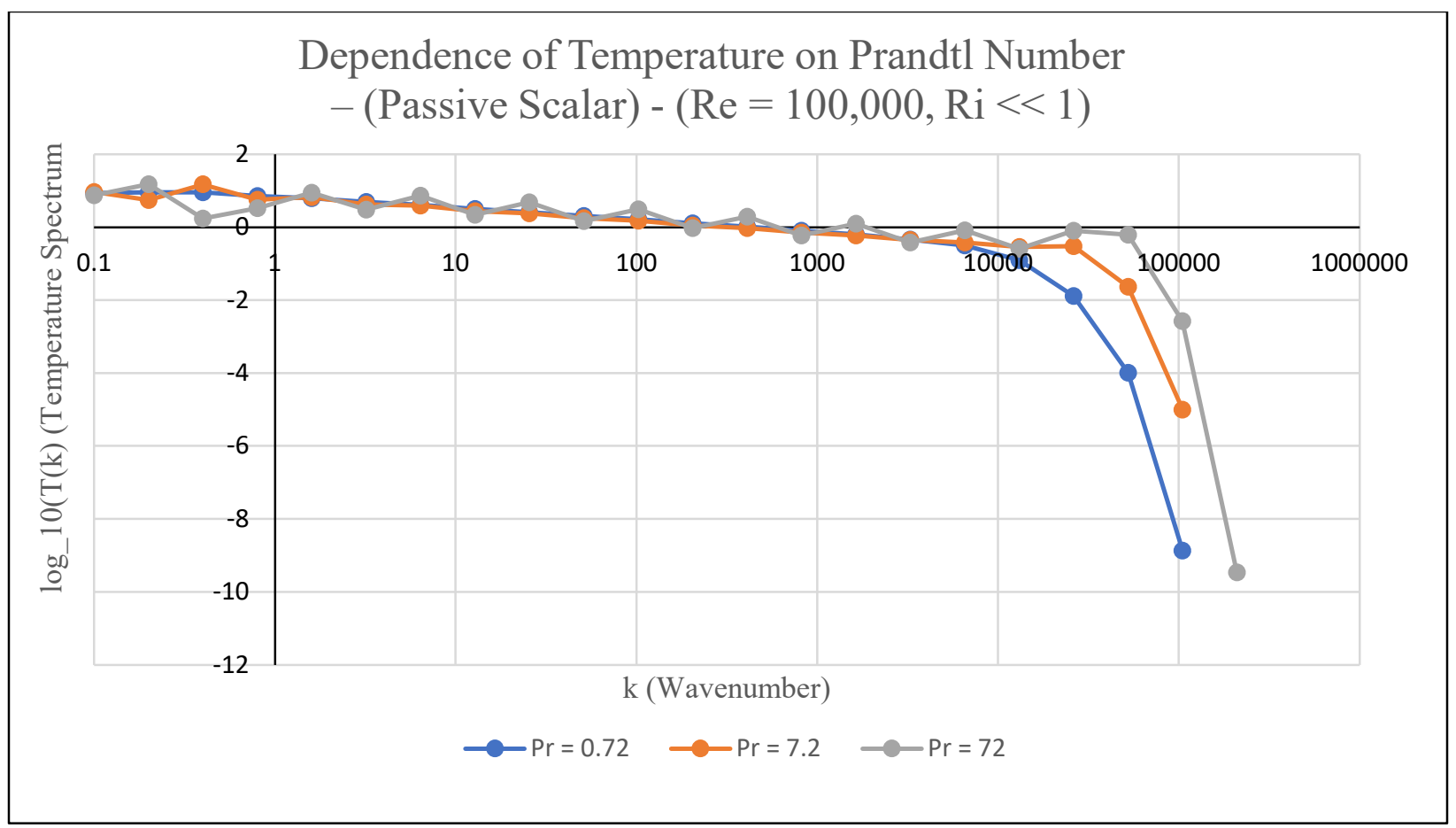

Figure 6. - Dependence of the Temperature Spectrum on Prandtl number.

Fig.6 shows dependence of temperature spectrum on Prandtl number. When the Prandtl number is increased, we started to observe numerical instability. For larger Prandtl numbers, we also see a bump forming near the beginning of the viscous-diffusive subrange of the turbulent cascade This bump is known as the "Hill" bump and it has been shown that appearance of the bump depends on the ratio between convective and diffusive energy transfer mechanisms. Thus the "Hill" bump is a function of the thermal diffusivity. Physically, we can think about the rate at which kinetic energy translates from the inertial subrange to the viscous-diffusive subrange of the cascade. There is some energy cascade rate at which this process occurs, and when the thermal diffusion is slower than this rate by some scalar factor, we started to see a build-up of the bump at small scales.

It was shown that the dyadic shell model predicts main properties of homogeneous isotropic turbulence for the range of non-dimensional parameters. An increase in the Reynolds number results in an increase in the size of the inertial subrange. Adapting the model from the 
temperature as a passive scalar to the active scalar does not affect the kinetic energy spectrum in a meaningful way due to the small value of the thermal expansion coefficient for air. Despite this, the shell-averaged entropy and the temperature pulsations both increase with time for the case of buoyancy driven turbulence.

\section{2 - Brandenburg Shell Model of Turbulence}

In this model we aim to simulate the effects of intermittency in the dissipative regime on the turbulent cascade. The general form of the Brandenburg shell model is as follows:

$$
\begin{gathered}
\frac{d u_{n}}{d t}=I_{u}\left(k_{n}\right)-v{k_{n}}^{2} u_{n}+\alpha g \theta_{n}, \\
\frac{d \theta_{n}}{d t}=I_{\theta}\left(k_{n}\right)-\kappa{k_{n}}^{2} \theta_{n}+\beta u_{n} \\
I_{u}\left(k_{n}\right)=a_{n} k_{n}\left(u_{n-1}{ }^{2}-2 u_{n} u_{n+1}\right)+b_{n} k_{n}\left(u_{n} u_{n-1}-2 u_{n+1}{ }^{2}\right), \\
I_{\theta}\left(k_{n}\right)=\tilde{a} k_{n}\left(u_{n-1} \theta_{n-1}-2 u_{n} \theta_{n+1}\right)+\tilde{b} k_{n}\left(u_{n} u_{n-1}-2 u_{n+1}{ }^{2}\right) .
\end{gathered}
$$

We want to make sure that the model satisfies theorem 4.1 as was done with the dyadic shell model, but the differences between temperature as active and passive scalar are not shown for the Brandenburg model. The effects of this forcing term were captured in the dyadic model and so for the rest of this discussion we consider temperature as the active scalar. The following two charts show the differences in the Dyadic shell model and the Brandenburg model for this case. 


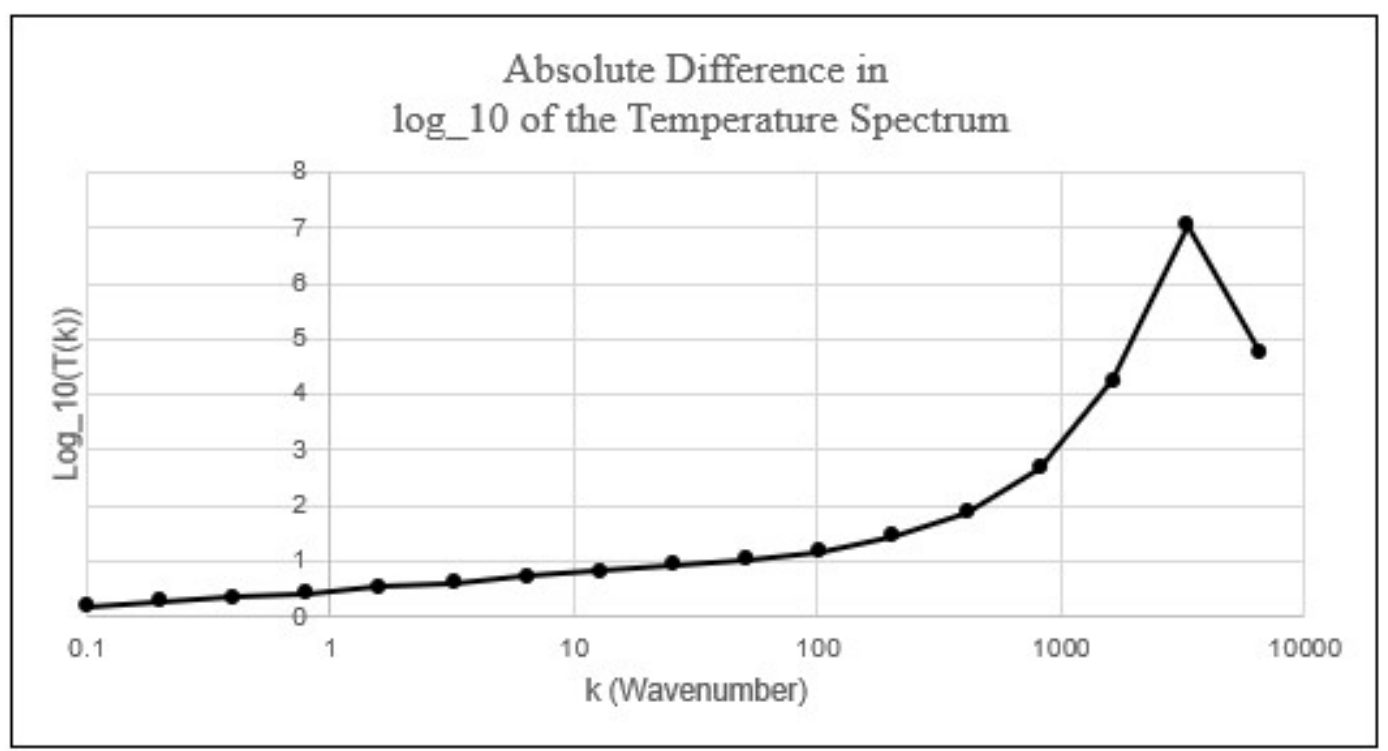

Figure 7. - Absolute Difference in $\log \_10$ of the Temperature pulsations for $\operatorname{Re}=400, \Delta \mathrm{T}=40$, $\operatorname{Pr}=0.72, \mathrm{Ri}<<1$

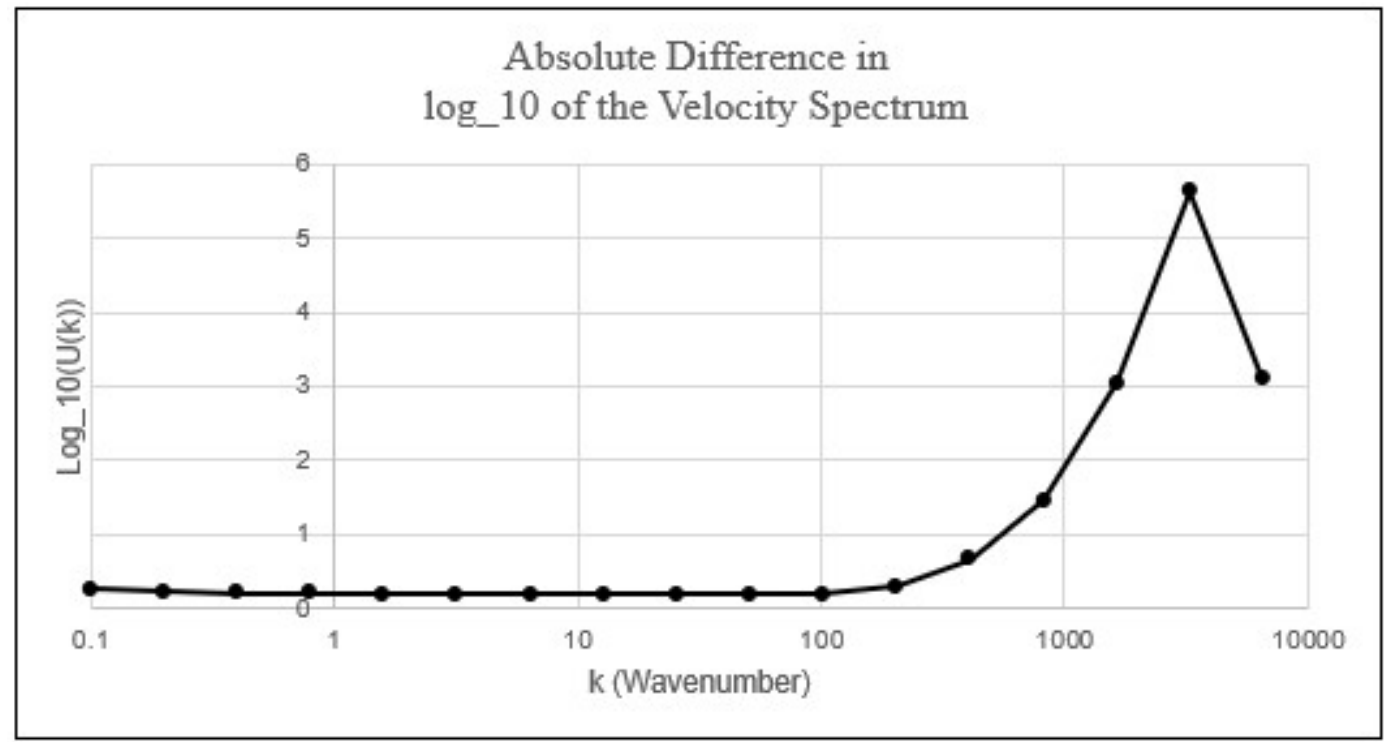

Figure 8. - Absolute Difference in $\log _{-} 10$ of the Velocity pulsations for $\operatorname{Re}=400, \Delta \mathrm{T}=40, \operatorname{Pr}=$ $0.72, \mathrm{Ri}<<1$

The increasing difference in the temperature pulsations level as we go towards the higher values of wave numbers indicates the influence of intermittency on the cascade behavior in the viscous-diffusive sub-range of the spectrum. The velocity pulsations are less affected but still impacted in a similar way. The intermittent behavior can be seen directly in the following chart showing an example of the burst in the energy dissipation rates with time. 


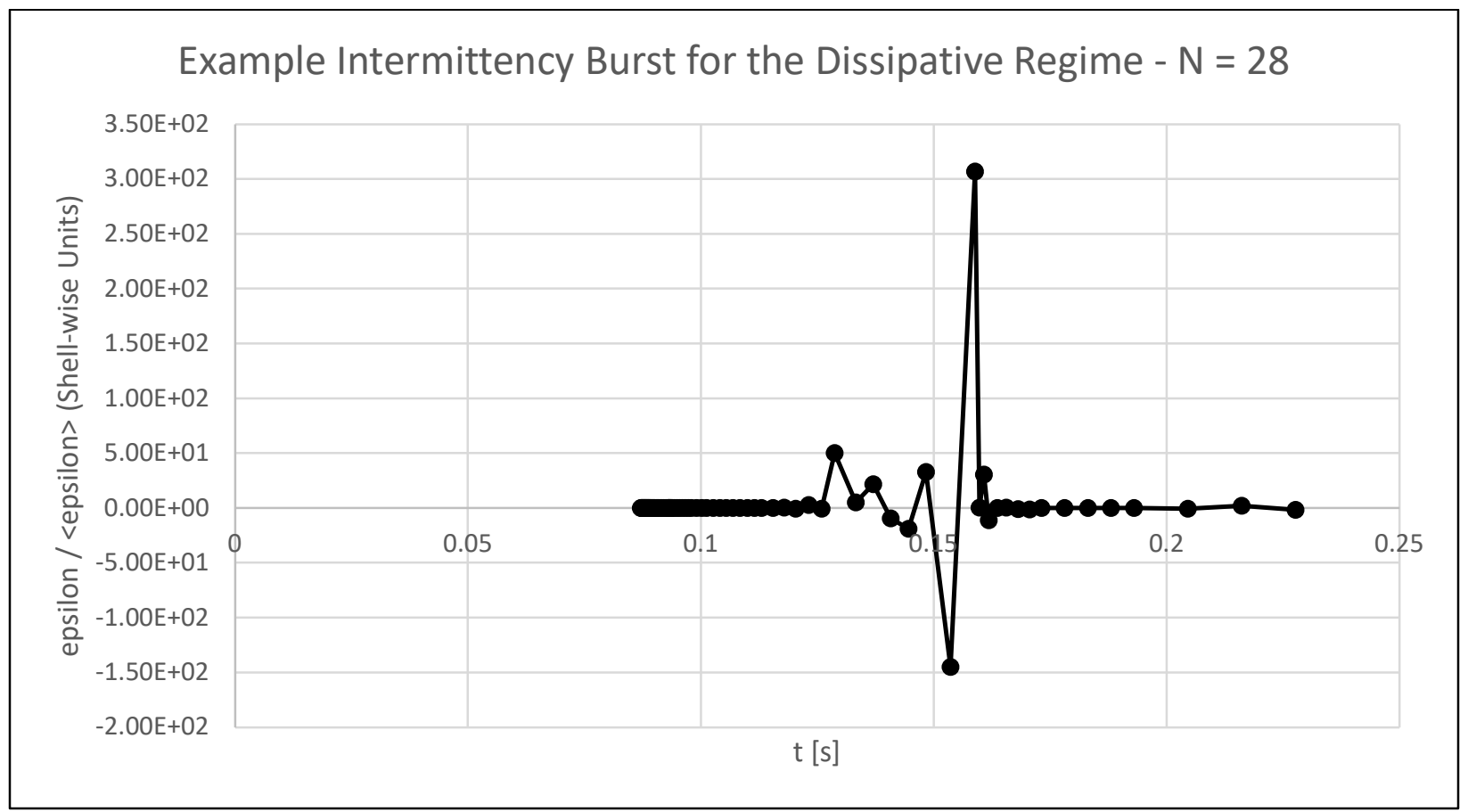

Figure 9. - Energy dissipation rate dependence on time (the Brandenburg Shell Model) 


\section{CHAPTER IV}

\section{LIGHT PROPAGATION IN A TURBULENT FLOW}

Physical theory of light propagation in different media is well studied but it is still not fully understood. In aero optics, we model the light as an electro-magnetic wave, rather than a collection of photons. Maxwell's equations describe the way in which the electric field is coupled to the magnetic field. This coupling is what causes the propagation of the electromagnetic wave in a vacuum. To understand propagation of light in a turbulent medium, we must first begin with a standard beam propagation through air. Light traveling through air experiences beam attenuation due to the interaction between the electromagnetic wave and the air particles. When the laser beam propagates in the turbulent atmosphere, we may observe additional scattering effects, which are caused by turbulent pulsations of velocity, temperature and pressure. One way to characterize this interaction is through the refractive index. The refractive index represents the phase velocity of light in the given medium relative to the speed of light in a vacuum. One can translate the refractive index of a fluid into density via the Gladstone Dale constant for the given fluid. When considering light propagation through turbulent medium, the general idea is to translate the temperature spectrum to the refractive index spectrum, which is highly non-uniform in this case. One important quantity to characterize is the wave front distortion of the light called the Optical Path Difference (OPD) which is understood through the difference in the Optical Path Length (OPL) of two different media.

$$
O P L=\oint n d s, O P D=s_{1} n_{1}-s_{2} n_{2}
$$

Many studies have studied the OPD for isotropic homogeneous turbulence. The flatwindowed cylindrical turret (FWCT) is a most common aero-optical system configuration, which 
is used to study aero-optical effects and the OPD [7]. Using commercial software CFD-FASTRAN the authors calculated the OPD values and Strehl Ratios, which is the ratio of the peak focal spot irradiance to the diffraction-limited peak irradiance, for standard isotropic turbulence.

In a study performed by J. Sontag et al, [8] experiments were conducted to determine the peak values for the root-mean square (rms) OPD. Experiments were done with the flow field Mach number around $\mathrm{M}=0.3$ using the Shack Hartmann wave front sensor to determine the wave frequency in which these values change in spatial and temporal modes. In general, they also determine a constant "A" which relates the Strehl ratio and the OPD measurements with the boundary layer thickness and local convective velocity profiles. The formula (25) shows the dependence of the " $\mathrm{A}$ " constant on a distance $\mathrm{y}$ in the direction perpendicular to the surface of the aircraft:

$$
A(y)=\frac{\theta_{r m s}(y)}{O P D_{r m s}(y)} \frac{U_{C}(y)}{U_{\infty}} \delta .
$$

For the considered cases, the value of $\mathrm{A}$ is 15 at the wall and decreases relatively linearly until $\mathrm{A}=5$ at $\mathrm{y} /=0.4$ where it begins to be constant for the remainder of the boundary layer. We can use the results from both [7] and [8] to determine an appropriate set of A values for shear layer turbulence.

These studies represent recent contributions to the aero-optical effects of wave propagation through a turbulent medium. However, the foundation of many results mentioned here is based on the fundamentals of the Tatarski theory.

\section{1 - Tatarski Theory and the Hill Bump}

The theory of laser propagation in turbulence was mainly developed by Tatarski [1]. This theory is based on first-principle fluid mechanics, electromagnetics, the mathematical theory of stochastic processes, and semi-empirical Kolmogorov and Obukhov-Corrsin similarity theories. 
The shell model (no forcing) and Tatarski theory predict that there will be no bump (Hill bump) prior to the viscous-diffusive subrange of the temperature spectrum. The shell model with forcing in the velocity pulsations equation at wavenumbers close to the transition to the dissipation subrange shows a bump. Fig. 10 shows the Hill bump capturing by different models for the correction functions applied to modify the Tatarski theory.

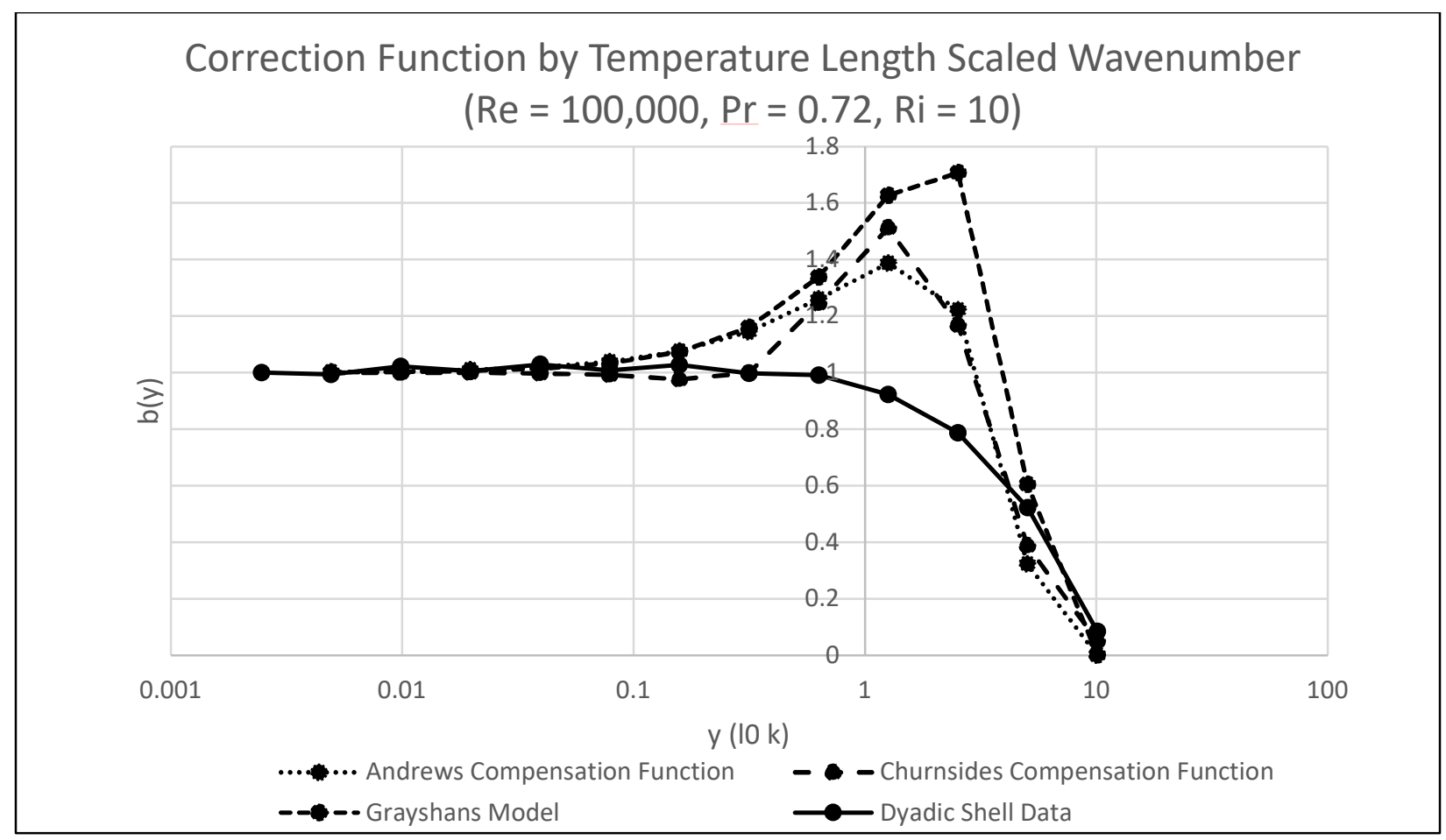

Figure 10. - Hill bump comparison to various existing similarity functions.

\section{2 - Irradiance Patterns and Split Step Method}

The propagation of the electromagnetic plane wave through turbulent media requires solution of the paraxial wave equation, derived from the Maxwell equations. While there are a variety of methods to do this, the split-step method is the most common used method, which assumes that a paraxial electromagnetic wave has a form

$$
E(z, t)=\varphi(z, t) \exp [-i \omega t]
$$




$$
\varphi(z, t)=A(z, t) \exp [i k z]
$$

It is assumed that $\mathrm{A}(\mathrm{z}, \mathrm{t})$ is a slowly varying scalar field of the phase amplitude. Thus, $\varphi(z, t)$ represents the phase of the electromagnetic wave. The following condition is then met: $\frac{\partial^{2} A}{d A^{2}} \approx 0$.

This can be plugged back into equation 26 and 27 and discretized by $h$ to get the following equation.

$$
A(z+h, r) \approx \exp \left[N \frac{h}{2}\right] \exp \left[\int L\left(z^{\prime}\right) d z^{\prime}\right] \exp \left[L \frac{h}{2}\right] \cdot A(z, r)
$$

The exponent in the middle is a representation of the accumulation of phase through the length $\Delta z$. Over this length we assume a constant phase screen generated by some Power Spectral Density of refractive index as shown below.

$$
\phi(x, y)=\sum_{n=-\infty}^{\infty} \sum_{n=-\infty}^{\infty} c_{n, m} \exp \left[i 2 \pi\left(f_{x_{n}} x+f_{y_{m}} y\right)\right] .
$$

Generally, the Fourier coefficients in (29) are collected from the Modified Von-Karman spectrum (30). This spectrum for the refractive index fluctuations is a common approach in the literature to reflect changes of the refractive index in a wave space.

$$
\Phi_{n}(k)=0.033 C_{n}{ }^{2} \frac{\exp \left(-\frac{k^{2}}{k_{m}^{2}}\right)}{\left(k^{2}+k_{O}^{2}\right)^{\frac{11}{6}}}
$$

where $k_{m}$ is the wavenumber corresponding to the inner scale of the turbulent spectrum; $k_{O}$ is the wavenumber corresponding to the outer scale of the turbulent spectrum.

Using this theory for the connection between turbulent characteristics and aero-optical effects requires knowledge of the size of the inertial sub-range of the turbulent spectrum. This can be taken from the model or through experimental hot-wire measurements.

The behavior of the model at large wave numbers $k$ is the key, for very small values of $k$ the chart rolls off to a constant. For large values of wave numbers, in the dissipative sub-range of 
the turbulent cascade, the model function is represented by an exponent as in the modified Tatarski theory. This model could be expanded further to include Hill bump effects by adding the special correction functions as was shown previously. 


\section{CHAPTER V}

\section{REFRACTIVE INDEX SPECTRUM}

The most critical part of this work is the connection of the shell models of turbulence with the theory of light propagation through turbulent medium. This connection made through the refractive index power spectral density (PSD). This PSD shows the strength of the refractive index fluctuations at each scale. Currently, the most popular method for getting this PSD is by using the Modified Von-Karman theory (MVK). MVK theory utilizes analytical functions for the energy containing scales, the inertial subrange, and the dissipative sub-range of the turbulent spectrum. The input parameters for this model, however, requires a knowledge of the largest and smallest

scales of the turbulence, which could be an issue. Furthermore, the effects of the Hill bump, intermittency, and non-linear effects of the model are not considered by these analytical functions. Our goal is to take the temperature spectrum given by the shell models and reconstruct the corresponding refractive index power spectral density.

\section{1 - Active Scalar Plots}

First, we will take the active scalar spectrum of temperature and construct the PSD using the relation between the refractive index spectrum and the temperature pulsations spectrum given by Jumper [3]. Once this is done, we can apply the split step method to construct relevant irradiance profiles as was done with the Modified Von-Karman PSD. 


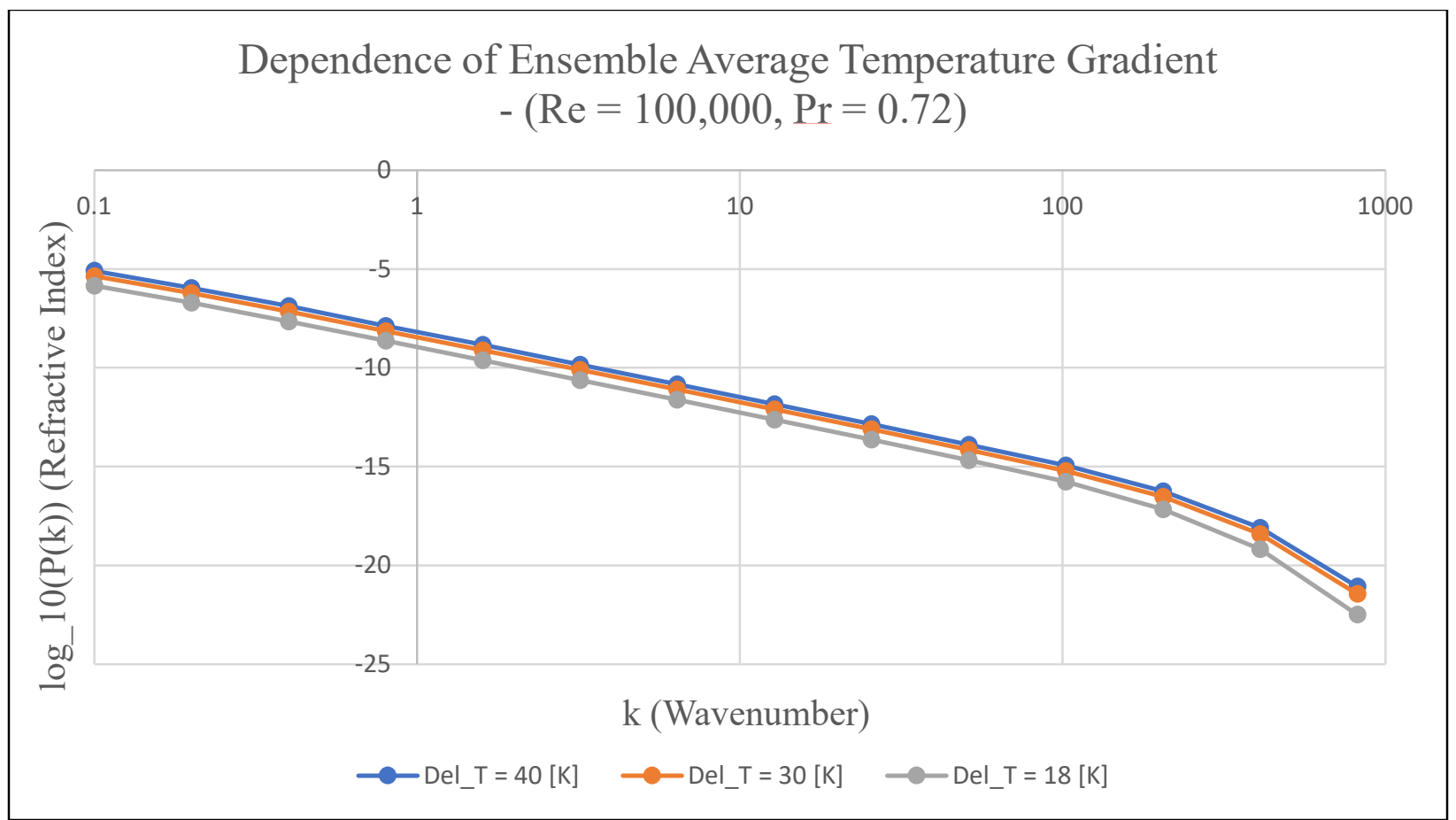

Figure 11. - Refractive Index Pulsation Spectrum Dependence on the temperature difference.

The figure above shows that the Power Spectral Density shifts up with an increasing temperature gradient coefficient. This means that with higher temperature differentials, the results of the shell models will be an increase in the strength of the turbulence properties.

\section{2 - Buoyancy Driven Turbulence}

The following irradiance profiles (Fig.12-13) show the refractive index power spectral density for the dyadic and Brandenburg shell models. 


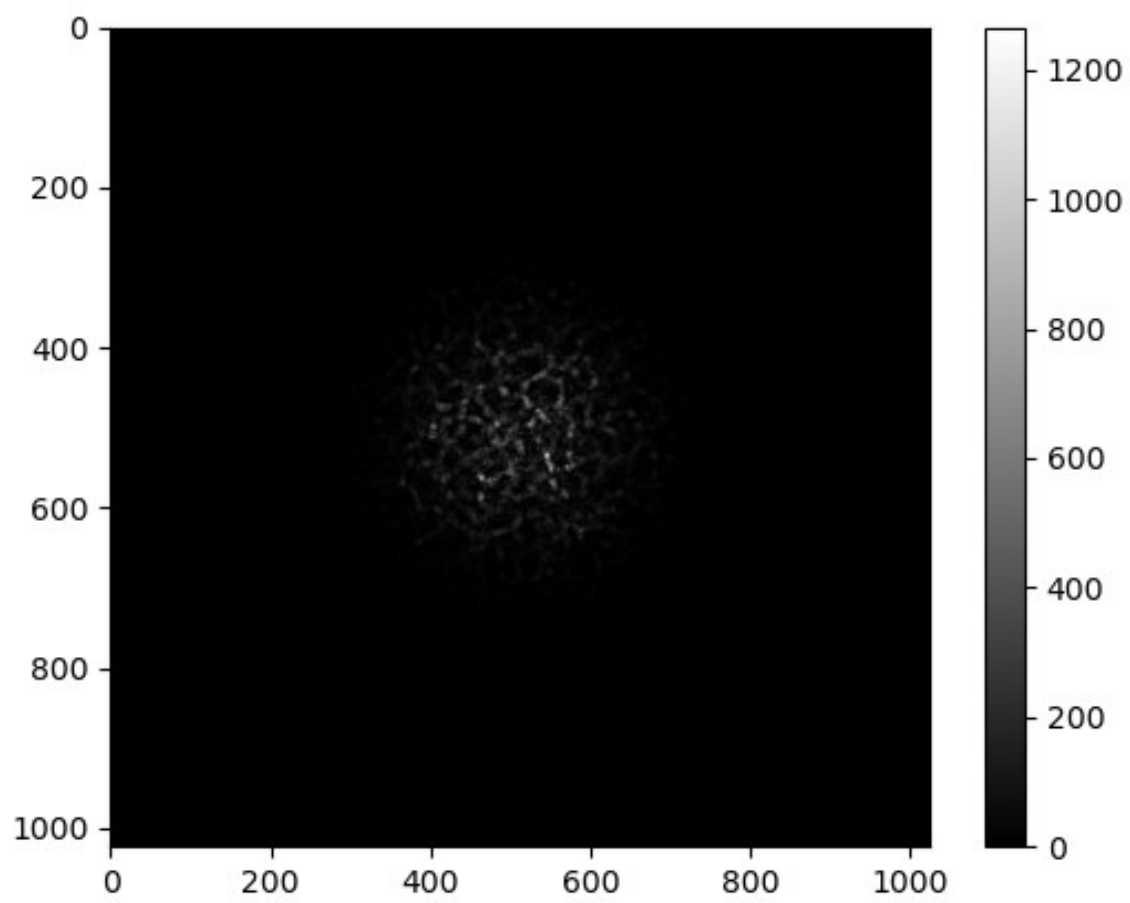

Figure 12. - Irradiance Profile generated using the temperature spectrum from the Dyadic Shell Model with $\operatorname{Re}_{\mathrm{L}} \sim 400$

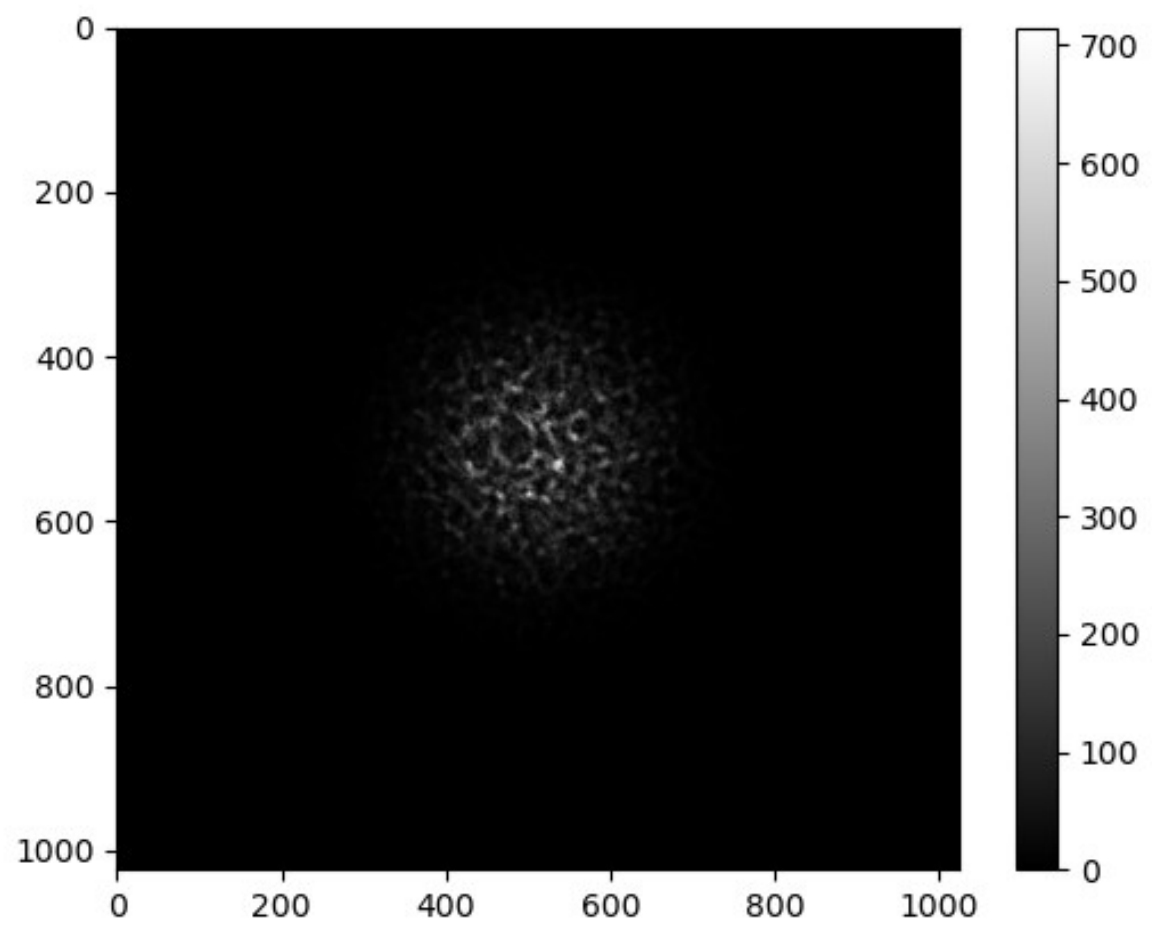

Figure 13. - Irradiance Profile generated using the temperature spectrum from the Brandenburg Shell Model with $\operatorname{Re}_{\mathrm{L}} \sim 400$ 
For buoyancy driven turbulence then, we started to see the effects of the reverse cascade and intermittency on the irradiance profile, showing that in this case there is a greater attenuation compared with the case of the pure direct cascade as in the dyadic shell model. There is also more beam spreading due to diffractive type effects. As the computational speed of solving these models is similar, it would be more advantageous to run a case for the Brandenburg model in practice.

\section{3 - Comparison to Experiments}

Generated irradiance profiles were compared to the experimental and DNS irradiance profiles. We can see that the strength of the fluctuations in each case vary, but the structures are similar for all three cases. The long structures are caused by local focusing, when light propagates through the turbulent region. One critical thing to note is that at short distances, refractive effects dominate. When moving further from the source, specifically in the Fraunhofer regime, diffractive effects became more important.
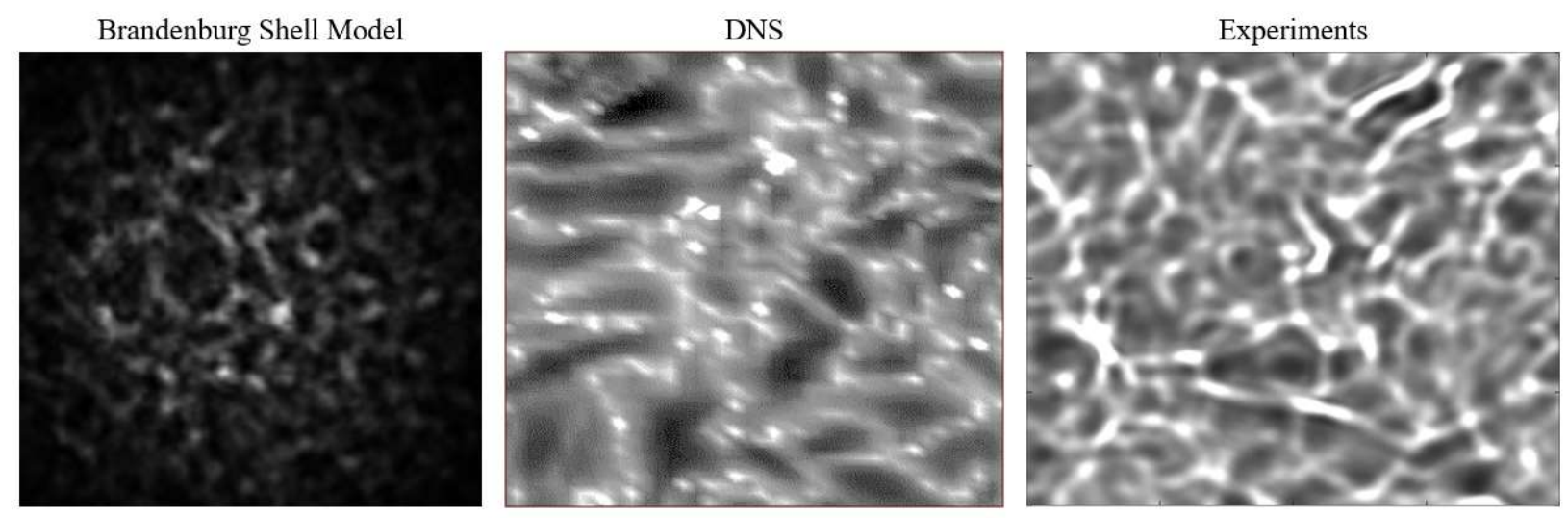

Figure 14. - Irradiance data visual comparison to DNS and experimental measurements (DNS -

(D. Donzis and K. Kumari, 9/1/2020), Experiment - (C. Limbach, 9/1/2020). 
Although this qualitative analysis may be effective for visualizing the physics of observed wave-front distortions, a detailed comparison of the statistical properties of turbulence producing these profiles is critical to understanding the results. To provide this analysis we used so called $\chi^{2}$ spectrum.

The $\chi^{2}$ spectrum in statistics is a sum of squares of $N$ gaussian distributed variables with zero mean and unit variance. In aero-optical applications the $\chi^{2}$ spectrum is introduced for the $\log$ amplitude of the power spectral density, which yields the following relation:

$$
\begin{gathered}
F_{\chi \chi}(k)=\pi k^{2} L\left(1-\frac{1}{\Lambda} \sin (\Lambda)\right) F_{n n}(k), \\
\Lambda=\frac{k^{2} L}{\kappa} .
\end{gathered}
$$

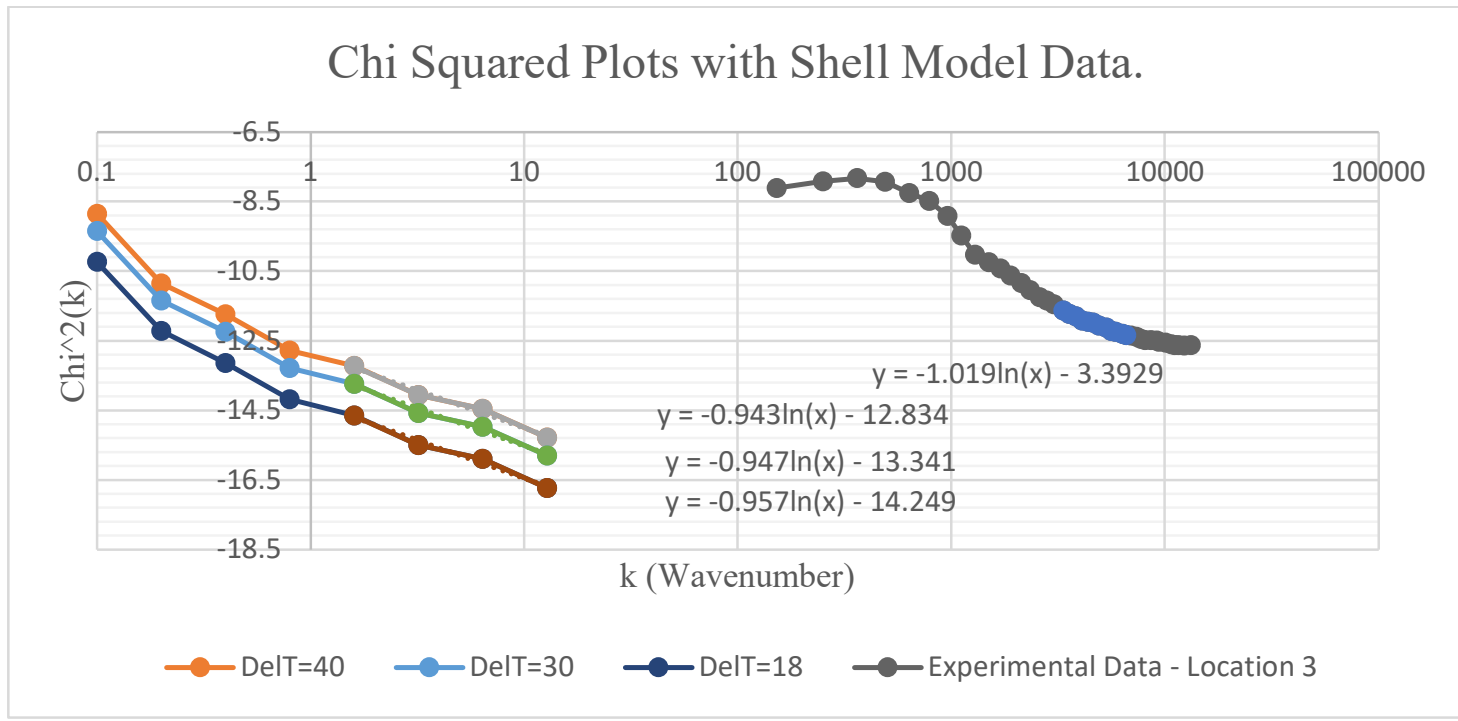

Figure 15. $-\chi^{2}$ statistics comparison between Shell Models and Experiments.

While the shell model has similar scaling to the experiment, we see that there are differences in the total energy on each scale. The shell model has less energy at smaller scales. This can be tuned with additional work on forcing terms for shell models. In the experimental spectrum, the initial values represent a developing turbulence which has anisotropic properties. Thus, we cannot expect, 
that the shell model developed for the case of isotropic turbulence will capture these effects. Once the turbulence is developed, the scaling is representative of what is captured in the model prior to the dissipative regime. 


\section{CHAPTER VI}

\section{CONCLUSIONS}

A direct connection was made between the shell models of turbulence, and low power beam propagation. For the parameter space containing the Richardson number, the Reynolds number, and the Prandtl number, we can get an irradiance profile that corresponds to these values directly. For buoyancy driven turbulence, an increase in the Reynolds number will result in the greater beam attenuation due an increase in the shell-wise temperature for a constant Prandtl number. The existing theory surrounding the Hill bump was extended to contain the effects from the dyadic shell model indicating that the bump appears when there is some additional forcing term before the dissipative sub-range of the spectrum [26-27]. According to the modeling results, the impact of the inverse cascade in combination with intermittent effects in the dissipative subrange of the spectrum cause more beam spreading and greater attenuation over the same propagation distance. Direct connection from the temperature spectrum generated by the shell models of turbulence to irradiance data of a beam propagating through isotropic turbulence has been performed. It was shown, that the $\chi^{2}$ statistics for the log amplitude of the power spectral density has similar scaling laws for both simulations and experiments but with different energy

levels at each scale which can be corrected with studies on forcing terms for shell models of turbulence. 


\section{REFERENCES}

[1] Tatarskii. "An analytical theory of pulse wave propagation in turbulent media.". Journal of Mathematical Physics. 20, 612. (1979).

[2] L. C. Andrews. "An analytical model for the refractive index power spectrum and its application to optical scintillations in the atmosphere". Journal of Modern Optics. Vol. 39, No. 9 1849-1853.

[3] E. Mathews, K. Wang, M. Wang, * E. J. Jumper. "The wavenumber spectra of aero-optical phase distortions by weakly compressible turbulence”. Optics Express. Vol 27. No. 4. Feb. 2019.

[4] Cheskidov, A., "Blow-up in finite time for the dyadic model of the Navier-Stokes equations," Trans. Am. Math. Soc. to be published.

[5] B. L. Sawford, P. K. Yeung, and J. F. Hackl, Reynolds number dependence of relative dispersion statistics in isotropic turbulence, Phys. Fluids 20, 065111 (2008).

[6] M. Obligado, M. Missaoui, R. Monchaux , A. Cartellier, M. Bourgoin (2011) Reynolds number influence on preferential concentration of heavy particles in turbulent flow. J Phys Conf Ser, ETC13, Warsaw, Poland: Sept 12-15, 2011 10.1088/1742-6596/318/5/052015

[7] M. Zhang, B. Shotorban, S. Zhang, Aero Optical Effects over a Cylindrical Turret, AIAA Journal.

[8] J. Sontag, S. Gordeyev, Spanwise Aero-Optical Measurements in a Subsonic Turbulent Boundary Layer to Study Velocity Field. University of Notre Dame, Indiana 
[9] Y. Diskin, M. Grose, M. Whitelet, K. Jackovitz, R. Drye, B. Hampshire, M. Owens, E.

Smith, E. Magee. Atmospheric and Aero Disturbance Characterization for DE System Applications.

[10] A. Roeder, D. Kurihara, H. Sakaue, S. Gordeyev. PSP Studies of the Wake Response Downstream of a Spanwise-Oscillating Hemispherical Turret. University of Notre Dame, Indiana.

[11] MIT Lectures on Turbulence. Chapter 5 Isotropic homogenous turbulence.

[12] M. Roberts, J. Bowman, B. Eckhart. Analytic Results from Shell Models of Turbulence. BIRS 16w5102: Recent Advances in Hydrodynamics.

[13] U. Frisch. "Turbulence the legacy of A.N. Kolmogorov" (1995)

[14] Cheskidov, A., Friedlander, S., Pavlović, N.: Inviscid dyadic model of turbulence: The fixed point and Onsager's conjecture. Journal of Mathematical Physics 48(6), 065503 (2007)

[15] A. A. Mailybaev. Continuous representation for shell models of turbulence. arXiv:1409.4682v1. [physics.flu-dyn]. 16 Sep. 2014.

[16] J. M. McDonough. Introductory Lectures on Turbulence. Department of Mechanical Engineering and Mathematics. University of Kentucky.

[17] Davidson, P.A. Turbulence: An Introduction for Scientists and Engineers. Oxford. 2004 [18] A. M. Obukhov, "The structure of the temperature field in a turbulent flow," Izv. Akad Nauk SSSR, Ser. Geogr. i Geofiz. 13, 58-69 (1949).

[19] “Split-Step Numerical Method.” Split-Step Numerical Method, www.simphotek.net/bckg/bckg.split-step.html. 
[20] Schmidt, Wolfram. Numerical Modeling of Astrophysical Turbulence. 2014. ISBN: 978-3319-01475-3.

[21] D. Donzis, K. Komal, Spectrum Normalization of log-amplitude $\chi$.

[22] G. Mendell. Introduction to signal processing and data analysis. LIGO Hanford Observatory. LIGO-G1200759

[23] L. Li, P. Liu, Y. Xing. H. Guo. Shell models for confined Rayleigh-Taylor turbulent convection. Commun Nonlinear Sci Numer Simulat 84 (2020). 105204

[24] Boussinesq, Joseph (1897). Théorie de l'écoulement tourbillonnant et tumultueux des liquides dans les lits rectilignes a grande section. 1. Gauthier-Villars. Retrieved 10 October 2015.

[25] A. N. Kolmogorov (1941). Local turbulence structure in incompressible fluids at very high Reynolds numbers. - Dokl. Akad. Nauk SSSR.

[26] R. J. Hill, “Models of the scalar spectrum for turbulent advection,” J. Fluid Mech. 88, 541-562 (1978).

[27] A. Muschinski. Temperature variance dissipation equation and its relevance for optical turbulence modeling. Journal of the Optical Society of America. Vol. 32, No. 11 . Nov. 2015.

[28] K. Kumari, D. Donzis. Irradiance profile from DNS simulation. 9/1/2020.

[29] C. Limbach. Irradiance profile from experiments. 9/1/2020. 